\title{
Erratum: Electron correlations in the antiproton energy-loss distribution in He [Phys. Rev. A 98, 012707 (2018)]
}

\author{
S. Borbély, X.-M. Tong, S. Nagele, J. Feist, I. Březinová, F. Lackner, L. Nagy, K. Tőkési, and J. Burgdörfer
}

(Received 5 June 2019; published 8 July 2019)

DOI: 10.1103/PhysRevA.100.019902

In our presentation of available data on straggling cross sections for protons and antiprotons in helium (Fig. 8), we have inadvertently omitted the experimental data by Besenbacher et al. [1] for protons and more recent improvements [2,3] of the original binary collision model [4], which now include screening and shell corrections. Therefore, the paper should be corrected as follows:

(1) Figure 8 and its caption are updated.

(2) The last paragraph on p. 9 should read as: "The analytic theory by Sigmund [4] also predicts a monotonic increase towards $T_{B}$ for $\bar{p}$ whereas for the charge-conjugate projectile $p$ this limit is approached from above and displays a peak around $20 \mathrm{keV}$. The peak is significantly reduced and shifted to higher projectile energies when effects of multiple Bohr oscillators and of shell and screening corrections are included in the binary theory formalism [2,3]. The enhanced straggling for $p$ originates from the combined effects of the Barkas contribution $\left(\sim Z_{p}^{3}\right)[7]$ and the charge-transfer channel absent for $\bar{p} . "$

(3) The list of references is amended.

None of the results and conclusions of the original paper are affected by these changes.

We thank Professor P. Sigmund for bringing this oversight to our attention and for providing us with the numerical data for the corrected binary theory.

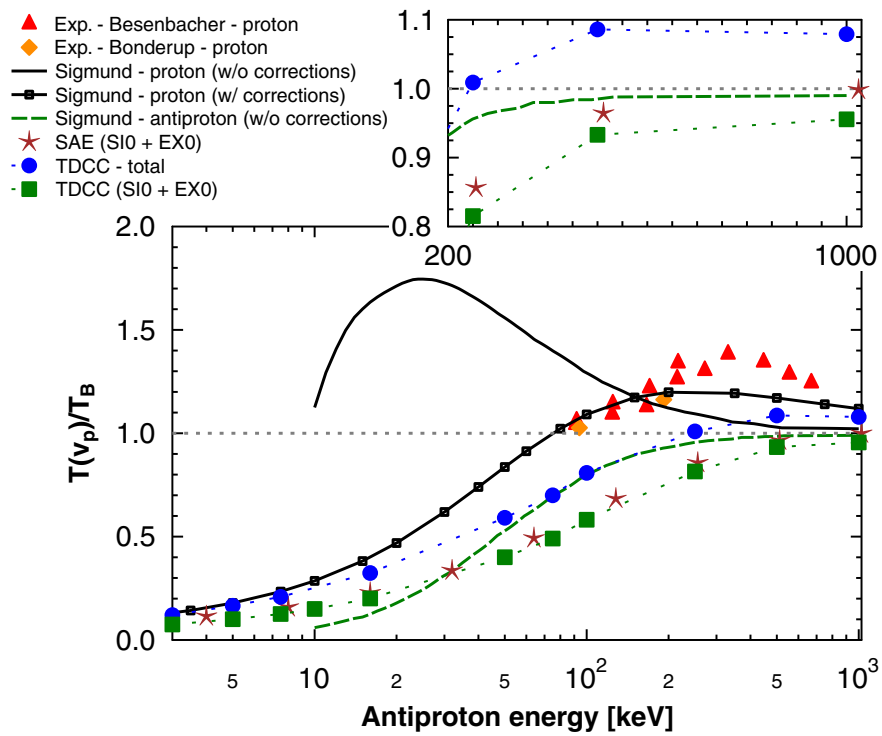

FIG. 8. Comparison between straggling cross sections for protons and antiprotons colliding with helium, normalized to the Bohr straggling number $T_{B}=4 \pi Z_{p}^{2} Z_{T}$. Shown are the present time-dependent close-coupling (TDCC) and single-active electron (SAE) results for antiprotons, the experimental data by Bonderup et al. [5] and by Besenbacher et al. [1] for protons. The analytic predictions by Sigmund for antiprotons and for protons are also shown, the latter both with [6] and without [4] corrections (see the text). The smaller frame is a zoom in on the high antiproton energy region showing the (non)convergence of the presented results towards the classical Bohr limit. 
[1] F. Besenbacher, H. H. Andersen, P. Hvelplund, and H. Knudsen, Mat. Fys. Medd. Dan. Vid. Selsk. 40, 1 (1981).

[2] P. Sigmund and A. Schinner, Eur. Phys. J. D 23, 201 (2003)

[3] P. Sigmund and A. Schinner, Eur. Phys. J. D 58, 105 (2010).
[4] P. Sigmund, Particle Penetration and Radiation Effects (SpringerVerlag, Berlin, 2006).

[5] E. Bonderup and P. Hvelplund, Phys. Rev A 4, 562 (1971).

[6] P. Sigmund (private communication).

[7] W. H. Barkas, J. N. Dyer, and H. H. Heckmann, Phys. Rev. Lett. 11, 26 (1963) 